\title{
Pre-eclampsia as a rare cause of severe hyponatraemia
}

\author{
Khyatisha Seejore ${ }^{1}$, Amal S. Mighell ${ }^{2}$, Alison J. Dawson ${ }^{1}$ \\ 'Department of Diabetes and Endocrinology, Bradford Teaching Hospitals NHS Foundation Trust, Bradford, UK \\ ${ }^{2}$ Maternity Services, Bradford Teaching Hospitals NHS Foundation Trust, Bradford, UK
}

\section{Introduction}

Hyponatraemia is a common electrolyte abnormality with multifactorial aetiology. It is associated with significant complications and carries a mortality rate of above $50 \%$ when plasma sodium concentration falls below $115 \mathrm{mmol} / \mathrm{L}$.

Pre-eclampsia toxaemia (PET) is a multisystem disorder that affects $2 \%$ to $5 \%$ of pregnancies and is responsible for up to $18 \%$ of maternal mortality. ${ }^{1}$ Classically, it is defined as hypertension and proteinuria $(\geq 0.3 \mathrm{~g}$ over 24 hours), with onset after 20 weeks gestation. It may also lower the threshold for seizures and predispose to foetal damage.

Severe hyponatraemia is a very rare, fatal complication of PET and has been described in only a few patients in the literature to-date. Here, we present a case of severe hyponatraemia complicating PET in a primiparous woman which resolved promptly postpartum.

\section{Case Report}

A 23-year old healthy primigravida was admitted at 34+6 weeks' gestation following an episode of reduced foetal movements. She was hypertensive (BP 171/98 $\mathrm{mmHg}$ ) and had 2+ proteinuria. Her sodium level was 133 $\mathrm{mmol} / \mathrm{L}$ (NR: 135-145mmol/L) and urine protein-creatinine ratio was 229 $\mathrm{mg} / \mathrm{mmol}$ (NR: 0-15). Cardiotocography was unremarkable. She was diagnosed with pre-eclampsia and started on labetalol and aspirin. She was discharged two days later with adequate BP control.

She was recruited into the PHOENIX trial, a multi-centre trial comparing outcomes between early induction (34-36+6weeks) versus expectant management (delivery: 37 weeks) in pre-eclampsia. She was randomised to the early induction arm and received IM betamethasone at 35+4 weeks' gestation. Serum sodium dropped to $126 \mathrm{mmol} / \mathrm{L}$ and two days later, reached a nadir of $114 \mathrm{mmol} / \mathrm{L}$ at $35+6 / 40$. She had now developed marked oedema and was admitted for further investigations, as outlined in Table 1.

Table 1: Summary of investigations as at $35+6$ weeks gestation

\begin{tabular}{|c|c|c|}
\hline Test & Value & Reference Range \\
\hline \multicolumn{3}{|l|}{ Urea and Electrolytes } \\
\hline Sodium & $114 * \mathrm{mmol} / \mathrm{L}$ & $135-145 \mathrm{mmol} / \mathrm{L}$ \\
\hline Potassium & $3.8 \mathrm{mmol} / \mathrm{L}$ & $3.5-5.0 \mathrm{mmol} / \mathrm{L}$ \\
\hline Urea & $8.3 * \mathrm{mmol} / \mathrm{L}$ & $2.5-7.8 \mathrm{mmol} / \mathrm{L}$ \\
\hline Creatinine & $62 \mathrm{umol} / \mathrm{L}$ & $49-90 \mathrm{umol} / \mathrm{L}$ \\
\hline \multicolumn{3}{|l|}{ Liver Function Tests } \\
\hline ALT & $721 * \mathrm{iu} / \mathrm{L}$ & $<40 \mathrm{iu} / \mathrm{L}$ \\
\hline Bilirubin & $21 \mathrm{umol} / \mathrm{L}$ & $2-21 \mathrm{umol} / \mathrm{L}$ \\
\hline Alkaline Phosphatase & $171^{*} \mathrm{iu} / \mathrm{L}$ & $30-130 \mathrm{iu} / \mathrm{L}$ \\
\hline Albumin & $21 * \mathrm{~g} / \mathrm{L}$ & $35-50 \mathrm{~g} / \mathrm{L}$ \\
\hline \multicolumn{3}{|l|}{ Thyroid Function Tests } \\
\hline Free T4 & $11.9 \mathrm{pmol} / \mathrm{L}$ & $10-20 \mathrm{pmol} / \mathrm{L}$ \\
\hline TSH & $1.6 \mathrm{miu} / \mathrm{L}$ & $0.2-4.0 \mathrm{miu} / \mathrm{L}$ \\
\hline \multicolumn{3}{|l|}{ Full Blood Count } \\
\hline Haemoglobin & $122 \mathrm{~g} / \mathrm{L}$ & $115-160 \mathrm{~g} / \mathrm{L}$ \\
\hline White Blood Cells & $12.0 \times 10^{9} / \mathrm{L}$ & $4-11 \times 10^{9} / \mathrm{L}$ \\
\hline Platelets & $248 \times 10^{9} / L$ & $150-400 \times 10^{9} / \mathrm{L}$ \\
\hline Random cortisol & $75 \mathrm{nmol} / \mathrm{L}$ (post IM betamethasone) & $150-600 \mathrm{nmol} / \mathrm{L}$ \\
\hline \multicolumn{3}{|l|}{ Urine } \\
\hline Urine osmolality & $445 \mathrm{mosm} / \mathrm{kg}$ & \\
\hline Urine sodium & $<10 \mathrm{mmol} / \mathrm{L}$ & \\
\hline Serum osmolality & $255^{*} \mathrm{mosm} / \mathrm{kg}$ & $275-295 \mathrm{mOsm} / \mathrm{kg}$ \\
\hline
\end{tabular}

Isotonic sodium chloride was carefully administered. She was delivered by caesarean section at $36+1$ weeks because of persistent hyponatraemia and worsening symptoms of pre-eclampsia as well as suspected acute fatty liver (ALT $1348 \mathrm{iu} / \mathrm{L} ; \mathrm{NR}<40 \mathrm{iu} / \mathrm{L}$ ).

A male infant was born (Apgar score 9 at 10 minutes) - he had mild hyponatraemia - corrected by the paediatricians. Within 24 hours of delivery, maternal hyponatraemia had improved to $133 \mathrm{mmol} / \mathrm{L}$. This is illustrated in Figure 1.

Recovery was complicated by intrapartum sepsis. She was discharged eight days later with a normal BP.

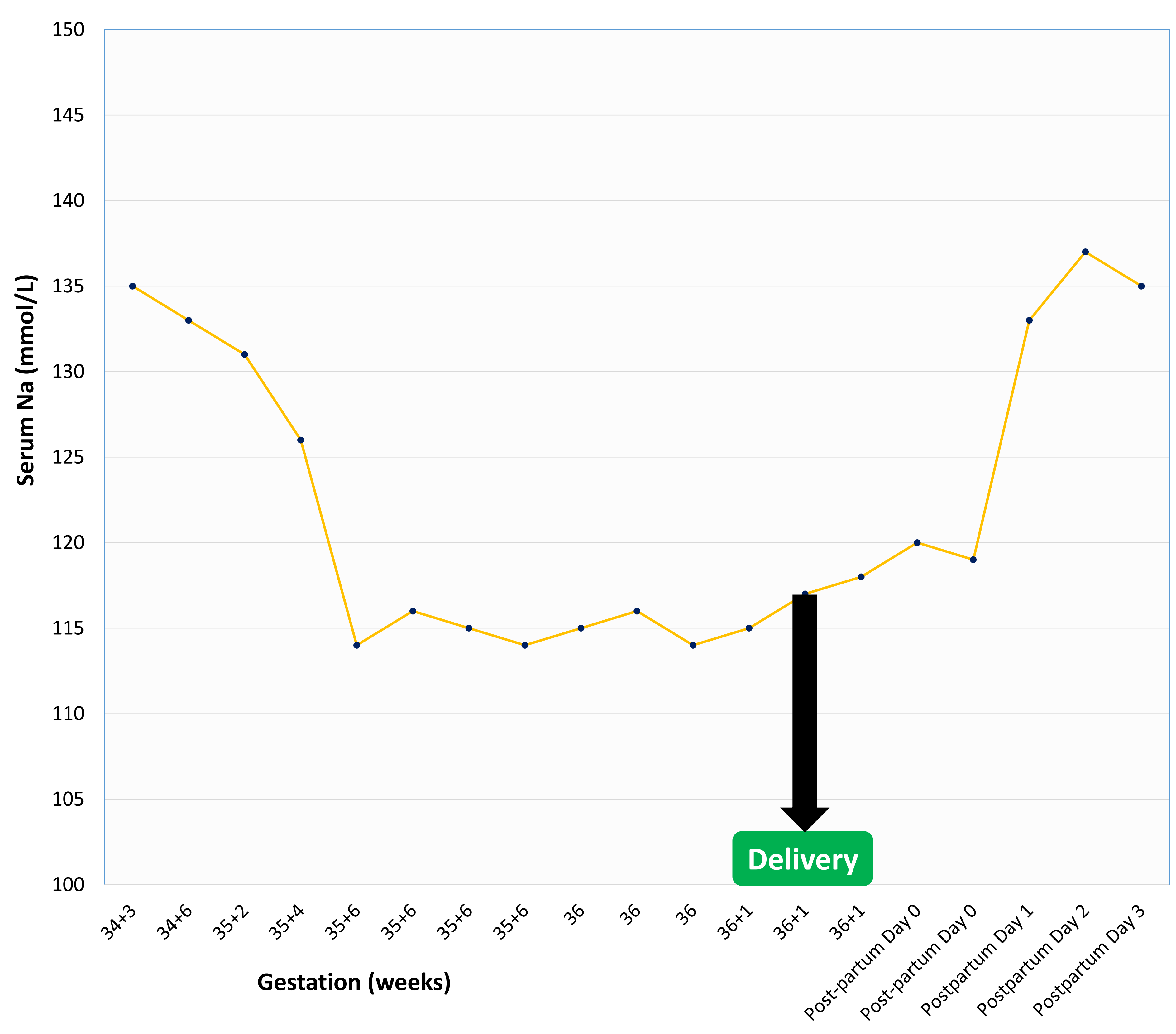

Figure 1: Variation in serum sodium level (NR: $135-145 \mathrm{mmol} / \mathrm{L}$ ) during admission and postpartum. There is a net improvement in serum sodium level after delivery at $36+1$ weeks gestation.

\section{Discussion}

Pregnancy involves physiological changes affecting water/ sodium homeostasis. However, most women with PET do not develop hyponatraemia. A recent review of 332 pregnancies complicated by PET found hyponatraemia to occur more frequently in older age and twin gestations; ${ }^{2}$ both features were absent in our patient. However, she had features of severe pre-eclampsia, including uncontrolled hypertension and impaired hepatic function.

We postulate that this was a case of hyponatraemia with hypervolaemia (excess extracellular sodium and total body water) as a result of impaired free water clearance secondary to pre-eclampsia. SIADH was discounted because of low urinary sodium and oedema.

We draw attention to severe hyponatraemia as a biomarker of severe preeclampsia and as a rare indication for urgent delivery. This requires multidisciplinary management and continuing postpartum care to ensure favourable maternal/ neonatal outcomes.

References:

1. Anglim B, Levins K, Bussmann N, et al (2016) Severe hyponatraemia associated with pre-eclampsia. BMJ Case Rep Published online: 10 August 2016 doi:10.1136/bcr-2016-215036

2. Razavi A.S., Chasen S.T., Gyawali R. et al. (2017) Hyponatraemia associated with pre-eclampsia. J. Perinat. Med., 45(4): 467-470 